\title{
Micrometer Accuracy Phase Modulated Radar for Distance Measurement and Monitoring
}

\author{
Sining An, Zhongxia Simon He, Member, IEEE, Jianguo Li, Jianping An, Member, IEEE and \\ Herbert Zirath, Fellow, IEEE
}

\begin{abstract}
An enhanced accuracy random binary phase modulated radar is proposed. It can be used in high accuracy monitoring in manufacturing. Compared with the traditional high accuracy radar using frequency modulated continuous wave (FMCW), the proposed radar system can be used in a multi-user scenario without occupying more bandwidth. A two-step distance estimation method is introduced to estimate the distance. First, the distance estimation accuracy is narrowed down to a half carrier wavelength by analyzing the envelope of the phase modulated signal. Then the carrier phase information increases the distance accuracy to several micrometers. An equalization method is introduced to solve the I/Q imbalance problem. The proposed radar system is demonstrated at a carrier frequency of $80 \mathrm{GHz}$ with a bandwidth of $2 \mathrm{GHz}$. The measured distance error was within $\pm 7 \mu \mathrm{m}$. In addition, a high measurement repetition rate of $500 \mathrm{kHz}$ was reached which is suitable for real-time monitoring in automatic manufacturing.
\end{abstract}

Index Terms - phase modulated, radar, millimeter-wave, micrometer accuracy, monitoring, Industry 4.0, distance measurement

\section{INTRODUCTION}

$\mathrm{T}$ major technology innovation trends, the fifth-generation network (5G) and industry 4.0, are undergoing revolutionary development. $5 \mathrm{G}$ is expected to start providing services in early 2020. 5G has three major performance targets: high speed (more than $1 \mathrm{Gbps}$ ), low power consumption and low latency (1 ms or less). 5G opens many new applications such as the massive internet of things, tactile internet, and robotics, etc. Industry 4.0, initiated as the promotion of manufacturing digitization, now extends to a broader concept including automation and data exchange in modern manufacturing technologies [1]. 5G technology supports Industry 4.0 by providing low power, low latency wireless communication infrastructure to connect all machines in a factory by exchanging massive data in real-time. The massive data which reflects the real operational status of machinery is obtained by different sensors.

This work was supported in part by the Swedish Foundation for Strategic Research (SSF) under Project SE13-0020 "High Datarate Wireless Communications (100G)" and in part by the Car2TERA project which has received funding from the European Union's Horizon 2020 research and innovation programme under grant agreement No. 824962.

S. An, Z. S. He (zhongxia@chalmers.se) and H. Zirath are with Chalmers University of Technology, Göteborg, Sweden. J. Li, J. An are with the Beijing Institute of Technology, Beijing, China.
There are many scenarios in automatic manufacturing which require high accuracy sensors. For example, to pick up and place very small and fragile objects, robotic arms require a special advanced sensing solution that can monitor position with micrometer accuracy. Moreover, monitoring in one direction is not always sufficient and multiple robotic arms need to work together in many cases [2]. Sensors need to be able to coexist with other sensors without interference. Compact radar sensors, that can measure the distance to a target with high accuracy, are crucial for the pick-and-place robotic arm operation in modern factories.

Traditional sensor distance measurement methods include ultrasonic, laser and radar. For the ultrasonic sensor, it is difficult to avoid interference when multiple sensors are closely installed. The laser requires a smooth surface on the target and a clear view, which are not viable in some applications. On the contrary, radar sensors can work in a multi-sensor scenario and do not require a smooth target surface and dust-free environment. Traditional FMCW radar uses a chirp signal which requires a certain bandwidth to achieve a high distance accuracy [3-5]. Typically, the FMCW radar cannot share the spectrum with other FMCW radars. With limited bandwidth, the number of FMCW radars that could coexist in the same area is limited. Interferometric radar uses only one continuous-wave (CW) signal, it can detect micrometer level object motion. However, its detection distance window equates to only one wavelength [6]. Using two tones, the unambiguity distance can be extended depending on the frequency gap between two tones [7-8]. For phase modulated radars, different coding can ensure multiple radars could coexist within the same spectrum slot. The accuracy of the phase modulated radar is however often limited by the modulation bandwidth [9].

In this paper, a phase modulated radar with micrometer accuracy is presented. Correlation-based time delay coarse estimation and carrier phase-based fine estimation are proposed to improve the distance accuracy to micrometer level with a measurement repetition rate of $500 \mathrm{kHz}$. The binary phase information also acts as a signature code which makes this radar system applicable in a multi-radar scenario where each radar has a different signature code. This paper is organized as follows. In section II, two radar principles are introduced with their advantages and disadvantages. The proposed phase modulated radar is introduced in section III with its operational principle and performance analysis. In section IV, the measurement setup and measurement results are presented. The conclusion and a comparison with other related works are given in section $\mathrm{V}$. 


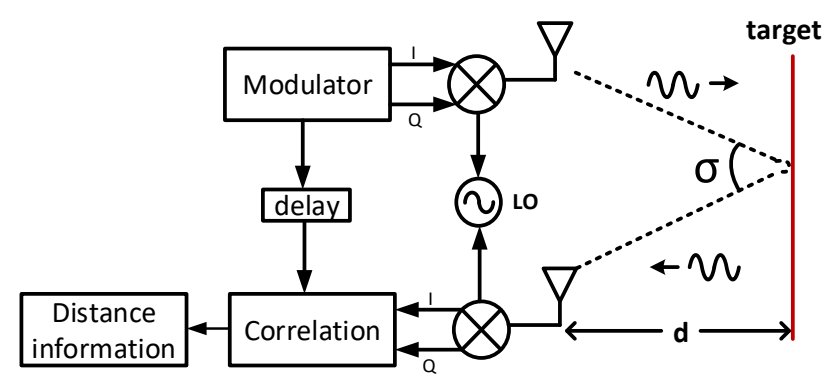

Fig. 1. The system structure of a RBPM radar.

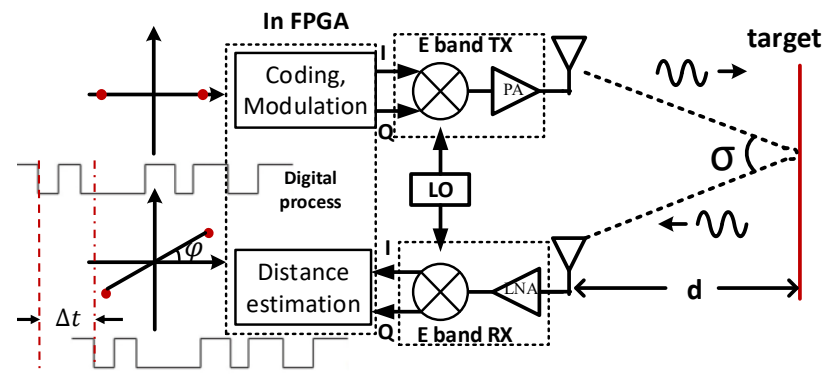

Fig. 2. Principle of the proposed radar system.

\section{TWO RADAR PRINCIPLES}

In this section, the traditional $\mathrm{CW}$ radar and random binary phase modulated (RBPM) radar are introduced. Their advantages and disadvantages are discussed.

In a traditional $\mathrm{CW}$ radar system, a $\mathrm{CW}$ signal is first generated by an oscillator and sent out by an antenna. After the reflected signal is received by the receiver antenna, the phase difference between the transmitted and reflected $\mathrm{CW}$ signal will be analyzed. From the phase difference $\varphi$ and the frequency $f_{c}$ of the $\mathrm{CW}$ signal, the distance $d$ between the radar and the target can be calculated by

$$
d=\frac{\varphi}{2 \pi} \times \frac{c}{f_{c}} \times \frac{1}{2} \times \cos \left(\frac{\sigma}{2}\right),
$$

where $c$ is the speed of light, $\sigma$ is the angle between the transmitted and reflected path. The accuracy of the distance depends on the phase accuracy of $\varphi$ and the carrier frequency $f_{c}$. With the same phase error $\varphi_{e}$, higher $f_{c}$ gives a lower distance error $d_{e}$. The unambiguous phase in a $\mathrm{CW}$ radar system is $2 \pi$, which means the maximum unambiguous distance a $\mathrm{CW}$ radar can detect is one wavelength. Using higher $f_{c}$ would give a shorter distance measurement of $\lambda=\frac{c}{f_{c}}$. As a result, most of the $\mathrm{CW}$ radars have high accuracy but short measurement distance.

On the other hand, RBPM radar has an advantage of long measurement distance but at the expense of lower accuracy [10-12]. Its system structure is shown in Fig.1. In this radar system, an up-converting mixer and a down-converting mixer are needed. The random binary sequence (RBS) is modulated to the baseband RBPM signal. This baseband signal is mixed with the LO signal by the up-converting mixer to radio frequency (RF) and is sent out by an antenna. The RF signal is reflected at the target and received by the receiver antenna. The reflected $\mathrm{RF}$ signal is down-converted to baseband by the down-converting mixer. A delay element and a correlator constitute a sliding correlator. The delay element is adjusted with a different time delay so that the transmitted baseband signal is correlated with the received signal with a different time delay.

The baseband RBPM signal $x(t)$ consists of two symbols, -1 and 1 . When $x(t)$ correlates with itself, the correlator outputs $\mathrm{L}$ as Eq. 2a shows; when $x(t)$ correlates with $x(t \pm \Delta t)$, where $\Delta t$ is larger than one symbol period, the correlator outputs a very low value around 0 as Eq. $2 \mathrm{~b}$ shows.

$$
\begin{aligned}
& \int_{t=0}^{L T_{\text {sym }}} x(t) \times x(t)=L, x(t)=\{-1,1\} \\
& \int_{t=0}^{L T_{\text {sym }}} x(t) \times x(t \pm \Delta t) \approx 0 \ll L, \Delta t \geq T_{\text {sym }}
\end{aligned}
$$

In Fig. 1, when the time delay from the delay element is equal to the time delay between the transmitted and reflected signals, the transmitted and the received signal are aligned, the correlator gives the highest output. By using this sliding correlator, the time delay between transmitted and reflected signal can be calculated with an accuracy of one symbol period.

The measurement distance $d_{\max }$ of this radar system is determined by the length of the random binary sequence (RBS) $L$ and the symbol rate $R_{s}$ as

$$
d_{\text {max }}=L \times \frac{C}{R_{S}} \times \frac{1}{2} \times \cos \left(\frac{\sigma}{2}\right) .
$$

For example, by using 1Gbaud RBS with a length of 128 symbols in the radar system, the maximum measurement of unambiguous distance is $38.4 \mathrm{~m}$. The distance accuracy is 30 $\mathrm{cm}$ in this case $\left(d_{e}\right.$ is within $\left.\pm 15 \mathrm{~cm}\right)$. By using a longer RBS, the measurement distance can be further increased. By increasing the symbol rate $R_{s}$, the distance accuracy can be further improved at the cost of using more bandwidth.

The CW radar has high accuracy but a short unambiguous distance. In contrast, the RBPM radar has low accuracy but a long measurement distance. By combining two radar principles, there is potential to achieve both high accuracy and long measurement distance in a radar system.

\section{Proposed Phase Modulated Radar System}

In this paper, an accuracy-enhanced RBPM radar is proposed with a two-step distance estimation method. The target distance can be expressed as

$$
d=(N+\varepsilon) \lambda,
$$

where $\lambda$ is the wavelength of the carrier signal, $\mathrm{N}$ is an integer number and $0<\varepsilon<1$.

A two-step estimation method is used in this paper, $\mathrm{N}$ is obtained during coarse estimation and $\varepsilon$ is calculated during fine estimation. In order to estimate $\mathrm{N}$ correctly, a special interpolation and correlation method is applied which allows target distance to be estimated with an accuracy of less than one carrier wavelength. For fine estimation, the phase offset of the carrier signal is then used to estimate $\varepsilon$ in Eq. 4. If $\varepsilon$ can be estimated with less than $0.1 \%$ error. This implies a distance accuracy of several micrometers when the carrier is at millimeter-wave frequency.

The proposed RBPM radar system is shown in Fig. 2. It comprises a millimeter-wave transmitter and a receiver, a shared local oscillator (LO) source, and two antennas. A pseudorandom binary sequence (PRBS) is modulated to a binary phase shift keying (BPSK) signal at baseband with a 
symbol rate of $R_{S}$ in the digital platform, a single piece of field-programmable gate array (FPGA). The BPSK signal is pulse-shaped and converted up to a carrier frequency $\omega_{c}$ using a direct conversion mixer. The signal propagates through the air until it gets to the target. The reflected signal travels back through the same path and is received by the receiver antenna. A receiver module down-converts the received signal to baseband BPSK signal. The baseband BPSK signal goes into the FPGA for digital distance estimation.

The transmitted BPSK signal can be represented as

$$
S_{t x}(t)=A e^{j \theta} e^{j \omega_{c} t}=A e^{j\left(\omega_{c} t+\theta\right)},
$$

where $\theta=\{0, \pi\}$. The received signal has a time delay compared with the transmitted signal. The received signal mixes with the LO signal, it becomes

$$
S_{r x}(t)=A^{\prime} e^{j\left[\omega_{c}(t-\Delta t)+\theta\right]} e^{-j \omega_{c} t} .
$$

After $S_{r x}(t)$ passes through a low pass filter with a bandwidth equal to the symbol rate $R_{S}$, the baseband signal is

$$
S_{r x}(t)=A^{\prime} e^{j\left(-\omega_{c} \Delta t+\theta\right)},
$$

where $\Delta t=\frac{2 d}{\operatorname{cocos}(\sigma / 2)}, d$ is the distance between the radar and the target. In the constellation diagram, the received baseband BPSK signal has a phase offset compared with the transmitted signal, as shown in Fig. 2. The absolute phase offset is

$$
\varphi+2 N \pi=-\omega_{c} \Delta t .
$$

The absolute distance from the radar system to the target can be calculated by

$$
d=(\varphi+2 N \pi) \times \frac{c}{2 \omega_{c}} .
$$

Here, $\mathrm{N}$ is the same as that of Eq. 4 , and $\varepsilon=\varphi / 2 \pi$. $\varphi$ can be observed from the constellation diagram. In the time domain, the time delay $\Delta t$ can be observed from the waveform of the transmitted and received signal, so that $\mathrm{N}$ could be estimated.

\section{A. Coarse estimation with full waveform matched filter}

As illustrated in Fig. 2, the received BPSK signal is time-shifted $\Delta t$ in comparison with the transmitted signal. The transmission signal is known to the receiver, therefore it is straightforward to estimate the time delay larger than a symbol period by using the sliding correlation method shown in Fig. 1. However, to correctly estimate $\mathrm{N}$ in Eq. 9, $\Delta t$ must be estimated with much higher accuracy within one carrier cycle period. For example, when $80 \mathrm{GHz}$ carrier frequency $\left(f_{c}\right)$ is used with 1 Gbaud $\left(R_{S}\right)$ BPSK signal, estimating $\mathrm{N}$ requires $\Delta t$ to be estimated with an accuracy higher than $R_{s} / f_{c}=1 / 80$ of the symbol period. However, the traditional RBPM signal radar has an accuracy of only one symbol period. To increase the accuracy, the sliding correlator needs to be able to provide a time delay with a step of $R_{s} / f_{c}=1 / 80$ of the symbol period which corresponds to one carrier cycle period. The correlation result with a different time delay needs to be able to be distinguished. In this case, a time delay with an accuracy of one carrier cycle period is obtained, $\mathrm{N}$ in Eq. 9 can then be correctly estimated. To achieve this goal, the baseband BPSK signal needs to be sampled with an impractically high sampling rate of 80 GSps. Furthermore, when the difference between the two delays is

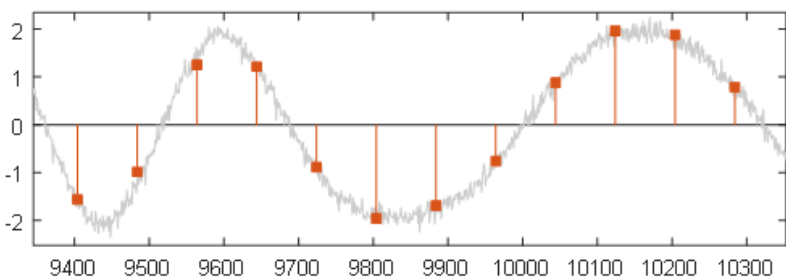

(a)

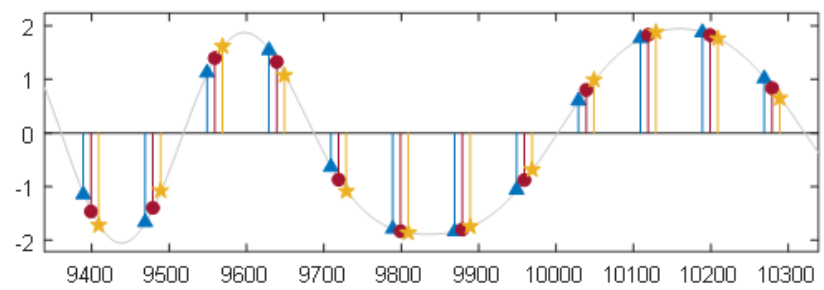

(b)

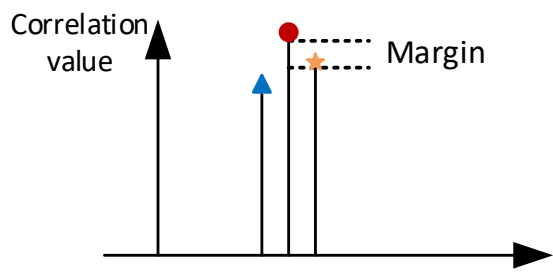

(c)

Fig. 3. (a) A typical reflected signal samples, (b) FWMFs, (c) Correlation result with different FWMFs.

smaller than one symbol period, the correlation results are similar. It is not straightforward to distinguish them. In our work, we proposed a matched filter-based estimation method that allows us to reach the desired estimation accuracy better than $1 / 80$ of one symbol period with only 2 GSps sampling rate.

The proposed radar system uses root raised cosine (RRC) pulse shaping to limit the bandwidth for a better signal-to-noise ratio (SNR). A typical received signal is illustrated in Fig. 3(a). Such a waveform is sampled at 2 GSps. An ideal transmission waveform consists of a full PRBS sequence $s(t)$ compared at the same sampling rate of $2 \mathrm{GSps}$ as the receiver. By moving the initial sampling position $\tau$ with a step of 1/80 symbol period, several groups of ideal sampling points are obtained as shown in Fig. 3(b) in different colors. Each group of points represents a full waveform matched filter (FWMF). The $i^{\text {th }}$ FWMF can be represented as:

$$
F_{i}[n]=\left.s(t)\right|_{t=\tau_{i}+n \times T_{s}},
$$

where $\tau_{i}=\frac{T_{c}}{2} \times i=i /\left(2 \times f_{c}\right)$ is the initial delay which increases by a half carrier period and $T_{S}$ is the sampling period as the signal digitized at the receiver with 2 GSps sampling rate.

With the received sampling sequence $r[n]$ as the input, the ith FWMF generates an output of the circular correlation of $r[n]$ and $F_{i}[n]$

$$
C_{i}(m)=\left|\sum_{n=0}^{L-1} r[n] \times F_{i}[[n+m]]_{N}\right|,
$$

where $L$ is the length of FWMF, $m$ is the sample offset from 0 to L. $F_{i}[[n+m]]_{N}$ is the circular shifted sequence of $F_{i}[n]$, where the $m$ symbols at the beginning of $F_{i}[n]$ move to the end of the sequence. With each $C_{i}(m)$, it only has two points that have 


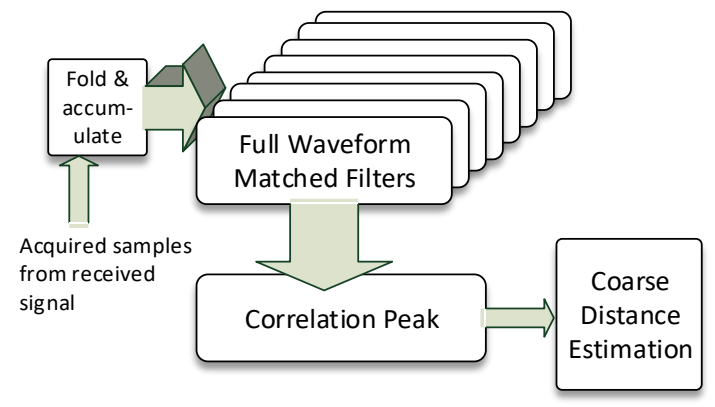

Fig. 4 Coarse estimation implementation flow diagram.

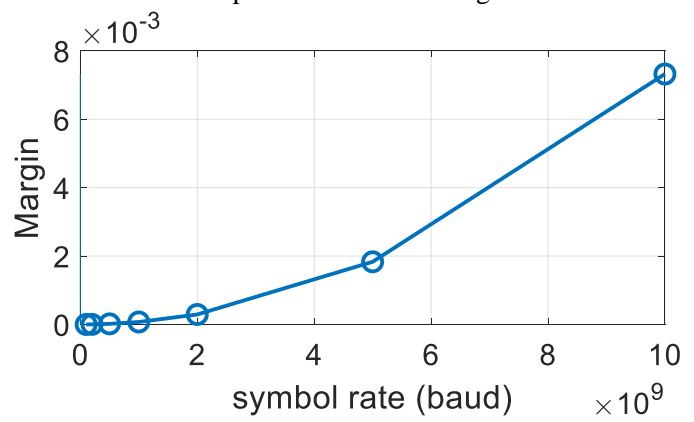

Fig. 5 Margin with different symbol rate.

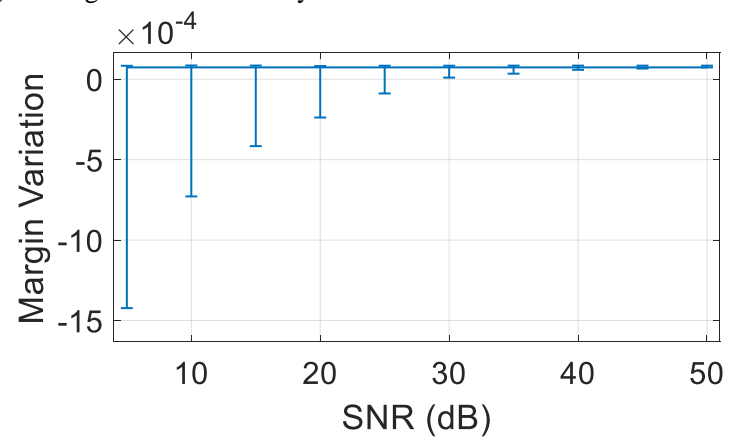

Fig. 6 The variance of margin with different SNR.

quite high values when $r[n]$ and $F_{i}[n+m]$ are aligned with the time offset smaller than one symbol period, the remaining points have very low values. Assuming that when $m=m_{\text {opt }}, C_{i}(m)$ has the highest value of $C_{i}\left(m_{o p t}\right)$, the estimated time delay between $r[n]$ and $F_{i}[n]$ is $m_{o p t} T_{s}$. Define the peak value of each FWMFs' output as

$$
P(i)=C_{i}\left(m_{\text {opt }}\right) .
$$

The highest peak value $P\left(i_{\text {est }}\right)$ among all FWMFs' output is generated when the $i_{\text {est }}{ }^{\text {th }}$ FMWF is most aligned with the received signal $r[n]$. As an example, passing the received signal $r[n]$ as shown in Fig. 3(a) through three FWMFs showed in different colored points in Fig. 3(b), the correlation peaks of three FWMFs' outputs are shown in Fig. 3(c). Naturally, we will decide the red FMWF (denoted as $i_{\text {est }}{ }^{\text {th }}$ FMWF) is the best estimation result since it generates the highest peak denoted as $P\left(i_{\text {est }}\right)$, and the neighboring FWMFs have lower peaks as $P\left(i_{\text {est }}-1\right)$ and $P\left(i_{\text {est }}+1\right)$. The time delay between transmitted and received signal is

$$
m_{o p t} T_{s}+\tau_{i_{\text {est }}}=m_{\text {opt }} T_{s}+i_{\text {est }} \times \frac{T_{c}}{2} .
$$

Fig. 4 shows the flow diagram of the coarse estimation implementation. First, from the acquired samples, the received data sequence needs to be folded and accumulated. At the transmitter side, the PRBS is repeatedly used in the transmitted signal. The FWMFs are sampled from the waveform with one PRBS chip (one chip includes a full-length PRBS). At the receiver side, if the sampling rate is an integer time of the PRBS chip rate and multiple PRBS chips have been acquired, the sampling positions in each PRBS chip are the same. As a result, the samples from different chips with the same sampling position can be accumulated to get a higher SNR. Then the accumulated signal passes through all FWMFs and the peak value of all FWMF output $P(i)$ are picked out to find the highest value among them $P\left(i_{\text {est }}\right)$. This indicates the $i_{\text {est }}{ }^{\text {th }}$ FWMF is most aligned with the received signal. From the peak information of this FWMF, the time delay between transmitted and received signal can be estimated by Eq. 13 .

When there is noise or interference in the system, the highest peak may indicate a wrong FWMF which will cause a wrong estimation. To study the robustness of this estimation method, we define a figure of merit as margin

$$
\zeta=\frac{P\left(i_{o p t}\right)-\max \left\{P\left(i_{o p t}-1\right), P\left(i_{o p t}+1\right)\right\}}{P\left(i_{o p t}\right)},
$$

where $P\left(i_{\text {opt }}\right)$ indicates the correlation peak value of the $i_{\text {opt }}{ }^{\text {th }}$ FMWF. The $i_{\text {opt }}{ }^{\text {th }}$ FMWF is aligned with the received signal $r[n]$. A high value of margin $\zeta$ implies higher confidence in the estimation. If a wrong estimation is made, a negative $\zeta$ would appear. Several practical aspects may affect margin $\zeta$, such as symbol rate, SNR, the length of the FWMF, the number of acquired samples and the sampling rate used in the receiver side.

First, we studied the margin $\zeta$ in relation to the transmission symbol rate. To achieve an accuracy higher than half of the carrier cycle period, $2 f_{\text {carrier }} / f_{\text {symbol rate }}$ number of FWMFs are needed to be used in the proposed radar system. Here $f_{\text {carrier }}=80 \mathrm{GHz}$. The estimation margin with no noise is plotted in Fig. 5, the margin increases as the symbol rate increases. Using a higher symbol rate in the radar system could increase the margin for the correct estimation. However, the higher symbol rate also implies higher bandwidth occupation.

Next, how the margin $\zeta$ varies with different SNR was also studied. Fig. 6 shows the variation of margin $\zeta$ with different SNR with 1 Gbaud symbol rate and 200 acquired samples (2 GSps sampling rate). The simulation was done 1000 times repeatedly to see how the margin $\zeta$ would vary. In Fig. 6, the vertical bar shows the variance of margin $\zeta$ with different SNR during 1000 times simulation. The horizontal bar on top is the Margin value without noise. As long as $\zeta$ is larger than 0 , it would give a correct estimation of the time delay. When there is noise in the system, $\zeta$ starts to dip below 0 which results in an incorrect estimation. It shows great potential to give a correctly estimated result for an SNR better than $30 \mathrm{~dB}$ with 1 Gbaud symbol rate.

In a noisy system, the variance of $\zeta$ can be reduced by acquiring more samples. The number of symbols $L$ in each FWMF is studied. FWMF can be designed with an arbitrary number of symbols. In a noisy system, increasing $L$ requires more hardware resources but gives more decision margin $\zeta$. Expected margin $\zeta$ and its variance are simulated versus 


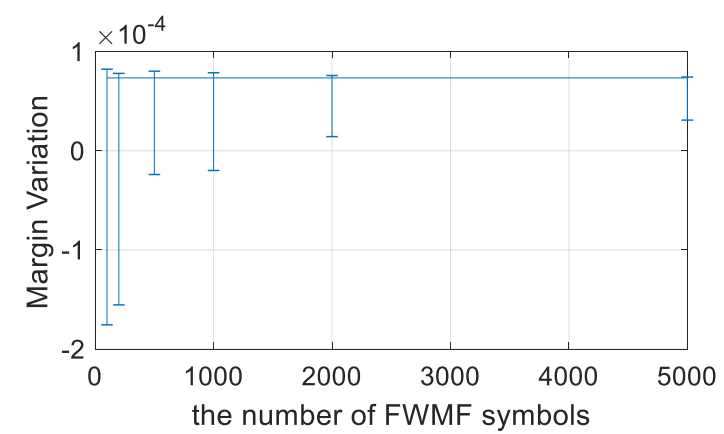

Fig. 7 The variance of margin with different length of FWMF.

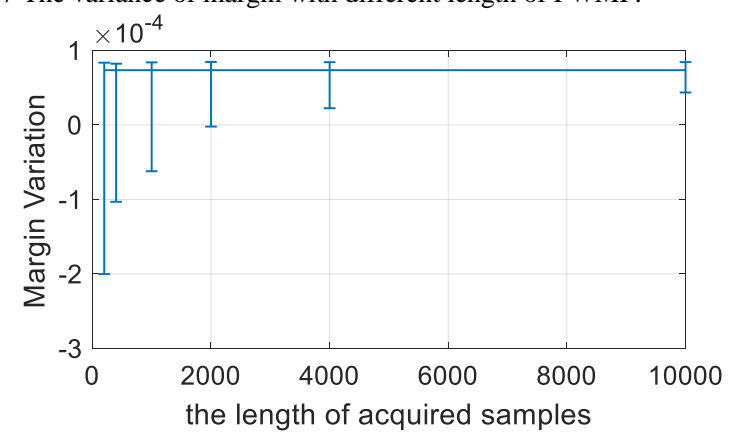

Fig. 8 The variance of margin with different length of acquired samples.

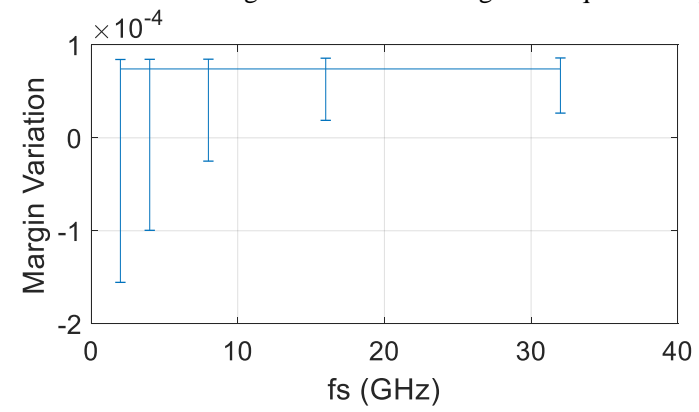

Fig. 9 The variance of margin with different sampling rate.

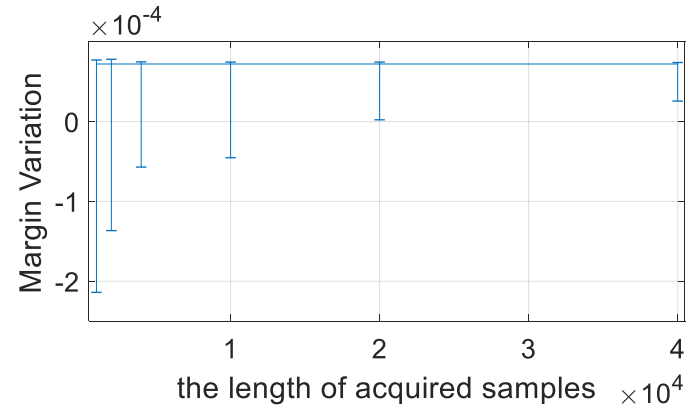

Fig. 10 The variance of margin with different length of acquired samples with the interference signal exist.

different FWMF length, assuming received SNR $=20 \mathrm{~dB}$, the symbol rate is 1 Gbaud, and the sampling rate is 2 GSps. The number of acquired samples equals $2 L$. Fig. 7 shows the simulated result of how margin varies with a different number of FWMF symbols. The simulation was repeated 1000 times. The variance of margin $\zeta$ decreases with the increase in the number of FWMF symbols. Using 2000 symbols in FWMFs, for 1000 simulations, gives $100 \%$ correct detection.

With a fixed number of symbols $L$ in FWMFs, acquiring more samples means the received signal will be folded and accumulated. The purpose of the folding is to increase the SNR. Fig. 8 shows the variance of $\zeta$ with a different number of acquired samples when SNR is $20 \mathrm{~dB}$. There are 100 symbols in FWMFs. The symbol rate is $1 \mathrm{Gbaud}$ and the sampling rate is 2 GSps. By acquiring more samples from 200 to 10000 , the variance of $\zeta$ becomes smaller. Using 4000 samples gives $100 \%$ correct estimation in 1000 simulations. As a result, the FWMFs can use fewer symbols to save on hardware resource.

Instead of increasing acquisition time, increasing sampling rate could also acquire more samples. Fig. 9 shows the variance of $\zeta$ with a different sampling rate when SNR is $20 \mathrm{~dB}$, the number of symbols L in FWMFs is 100 and the number of acquired symbols is also 100 . The simulation was repeated 1000 times. With the sampling rate increased, the variance of margin $\zeta$ is decreasing, and the estimation is more robust. When increasing the acquiring time or using a higher sampling rate ADCs can help to acquire more samples with a tradeoff of lower measurement repetition rate or higher hardware cost, respectively.

\section{B. Multi-user Interference Resistance}

Using the PRBS in the radar system provides an advantage of strong resistance to multi-user interference. When correlating two different PRBS, the correlator will output a very low value around 0. Only if the two input signals use the same PRBS, will the correlator output a very high value.

In the multi-user scenario, each user uses a unique PRBS. In each radar system, the receiver may receive an interference signal from another user nearby. Since users are using different PRBS, the interference signal only acts as additive noise. As discussed in the previous section, the estimation accuracy can be increased by acquiring more samples. Fig. 10 shows the variance of margin with different length of acquired samples when there is an interference signal. The power ratio between the signal and the interference is $10 \mathrm{~dB}$, the sampling rate is 2 GSps, the symbol rate is 1 Gbaud. When the number of acquired samples is more than 20000 , the margin remains positive during 1000 simulations. Even the noise power increases, this radar system still works by acquiring more samples.

\section{Fine estimation with carrier phase analysis}

Similar to the $\mathrm{CW}$ radar, the fine distance within one carrier wavelength can be estimated from the phase offset of the carrier. However, in a practical radar system, there are some challenges to get the correct phase. For the $\mathrm{CW}$ radar, the reflected $\mathrm{CW}$ signal needs to mix with two $\mathrm{CW}$ signals from the transmitter side. These two signals have a phase difference of 90 degrees. After mixing and passing through a low pass filter (LPF), there will be two output values representing $\cos (\varphi)$ and $\sin (\varphi)$, respectively. Then $\varphi$ can be calculated from these two values. However, if there is I/Q imbalance between two CW signals, they do not have a 90-degree phase difference, then the values of $\cos (\varphi)$ and $\sin (\varphi)$ will be inaccurate. For the proposed RBPM radar, the $\mathrm{I} / \mathrm{Q}$ imbalance is introduced in the receiver module.

When there is no imbalance, with different $\varphi$, there is a circle of dots in the constellation diagram of $\cos (\varphi)+j \sin (\varphi)$. When there is I/Q imbalance, the circle becomes an oval shape. This imbalance can be removed with the equalization function

$$
y(n)=x(n)+(\alpha+j \beta) x(n)^{*},
$$




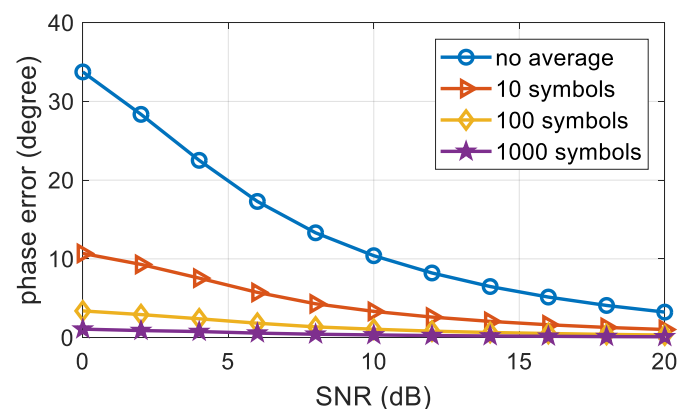

Fig. 11 The phase error with different SNR and different numbers of symbols for average when there is no interference.

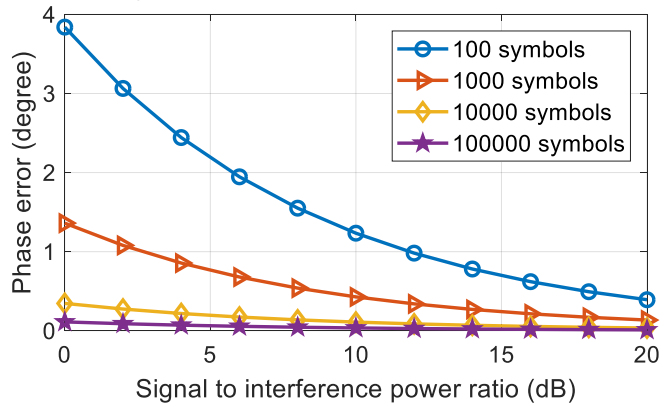

Fig. 12 The phase error with different signal to interference power ratio and different numbers of symbols for average when there is no noise.

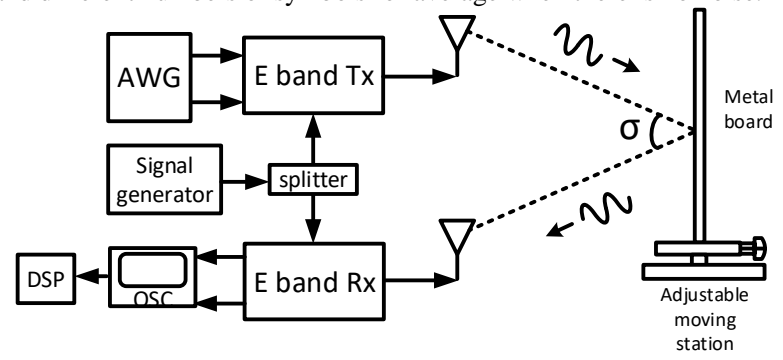

Fig. 13. Measurement set-up.

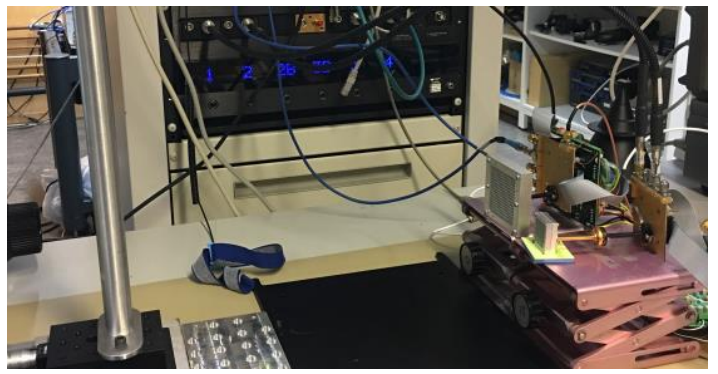

Fig. 14 The measurement setup in lab.

where $x(n)=\cos (\varphi)+j \sin (\varphi), x(n)^{*}$ is the conjugate value of $x(n) . \alpha$ and $\beta$ are two parameters that adjust the imbalanced signal $x(n)$ [13-14]. By tuning these two parameters, the imbalance can be removed, then constellation points will form a circle again.

Another challenge in the practical radar system is the noise and interference which would result in inaccurate $\varphi$ estimation. This problem can be solved by averaging multiple symbols' phases. For the proposed RBPM radar, the received baseband signal is

$$
S_{r x}(n)=A^{\prime} e^{j[\varphi+\theta(n)]}+A^{\prime \prime} e^{\left(j \varphi_{i}+\theta_{i}(n)\right)}+N_{0}(n) .
$$

where $\theta(n)=\{0, \pi\}, A^{\prime \prime} e^{\left(j \varphi_{i}+\theta_{i}(n)\right)}$ is the interference signal, $N_{0}(n)$ is the noise. Using the transmitted signal to correlate with the received signal, the correlation result is

$$
\begin{aligned}
P & =\sum_{n=1}^{K}\left[A^{\prime} e^{j[\varphi+\theta(n)]}+A^{\prime \prime} e^{j\left[\varphi_{i}+\theta_{i}(n)\right]}+N_{0}(n)\right] A e^{j \theta(n)} \\
& =A A^{\prime} K e^{j \varphi}+\sum_{n=1}^{k}\left[A A^{\prime \prime} e^{j\left(\varphi_{i}+\theta_{i}(n)+\theta(n)\right)}+N_{0}(n) e^{j \theta(n)}\right],
\end{aligned}
$$

where $\sum_{n=1}^{k} N_{0}(n) e^{j \theta(n)} \approx 0$. Since $\theta_{i}(n)$ and $\theta(n)$ are two different random sequences,

$$
\sum_{n=1}^{K} e^{j \theta_{i}(n)} e^{j \theta(n)} \approx 0 .
$$

The averaged phase can be calculated as

$$
\varphi_{\text {ave }} \approx \operatorname{angle}(P) \text {. }
$$

With $\mathrm{K}$ increased, the effect of noise and interference becomes smaller. Fig. 11 shows the phase error of $\left|\varphi-\varphi_{\text {ave }}\right|$ with different SNR when there is no interference signal. With an increasing number of SNR and the symbols used for phase average, the phase error decreases. Fig. 12 shows the phase error with different signal to interference power ratios when there is no noise. The phase error is reduced by using more symbols for the average.

\section{EXPERIMENTAL VERIFICATION}

\section{A. Experimental Setup}

The laboratory experimental setup is shown in Fig. 13. Integrated E-band transmitter and receiver modules are used to generate and receive a signal at $80 \mathrm{GHz}$. A metal bar is used as the target located $60 \mathrm{~cm}$ away from the antennas. An arbitrary waveform generator (Keysight M8195A) is used for generating a BPSK modulated signal of 1 GBaud with RRC pulse phasing, and a real-time oscilloscope (Teledyne LabMater $10 \mathrm{Zi}-\mathrm{A}$ ) is used to capture the received signal, and data is processed off-line using Matlab. A pair of E-band transmitter and receiver (Gotmic gTSC0023 \& gRSC0015) are used in this system. They are sharing the same local oscillator (Agilent E8257D) and therefore fully phase synchronized. The received quadrature baseband signals are sampled by the oscilloscope at a sampling rate of $2 \mathrm{GSps}$. Both coarse and fine estimation are performed as mentioned in section III. A picture of the measurement setup in the lab is shown in Fig. 14.

\section{B. Coarse estimation with full waveform matched filter}

The target is placed $60 \mathrm{~cm}$ away from the antennas, and its position is moved with a manual micrometer positioner from this initial position with $1 \mathrm{~mm}$ steps away from the radar. The radar uses $80 \mathrm{GHz}$ carrier frequency and repeatedly sends a PRBS with a length of 2000 symbols. The roll-off factor of the RRC pulse shaping filter is 0.5 . The received baseband signal is sampled by an oscilloscope at 2 GSps and the offline process is performed using the algorithm proposed in section III. A.

The processed result of the coarse estimation is plotted in Fig. 15, where the $\mathrm{x}$-axis is the target distance away from the initial position. The $y$-axis is the estimation error with the millimeter unit. The accuracy threshold of the coarse estimation is $\pm 1.875 \mathrm{~mm}$ (half of the wavelength in air of $80 \mathrm{GHz}$ ). 4000 samples were used for coarse estimation. As shown in the figure, the measurement shows max error less than $1.5 \mathrm{~mm}$ thus 
TABLE I

SUMMARY OF TYPOGRAPHICAL SETTINGS

\begin{tabular}{|c|c|c|c|c|c|c|c|}
\hline Ref. & $\begin{array}{c}\text { Freq. } \\
(\mathbf{G H z})\end{array}$ & Bandwidth & Method & $\begin{array}{c}\text { Accuracy } \\
(\boldsymbol{\mu} \mathbf{m})\end{array}$ & $\begin{array}{c}\text { Target to radar } \\
\text { Distance }\end{array}$ & $\begin{array}{c}\text { Measured } \\
\text { Distance }\end{array}$ & $\begin{array}{c}\text { Unambiguity } \\
\text { Distance }\end{array}$ \\
\hline$[3]$ & 122.5 & $1 \mathrm{GHz}$ & FMCW & \pm 2 & $2 \mathrm{~m}$ & $5 \mathrm{~mm}$ & $>1 \mathrm{~km}$ \\
\hline$[4]$ & 124 & $6 \mathrm{GHz}$ & FMCW & \pm 6 & $1.6 \mathrm{~m}$ & $35 \mathrm{~mm}$ & $>1 \mathrm{~km}$ \\
\hline$[5]$ & 80 & $10 \mathrm{GHz}$ & FMCW & \pm 0.5 & $0.5 \mathrm{~m}$ & $2 \mathrm{~mm}$ & $>1 \mathrm{~km}$ \\
\hline$[6]$ & 24 & Single tone & Interferometer & \pm 0.5 & $1 \mathrm{~m}$ & $10 \mu \mathrm{m}$ & $12.5 \mathrm{~mm}$ \\
\hline$[7]$ & 24 & Two tone & 2-tone Interferometer & \pm 200 & $1 \mathrm{~m}$ & $60 \mathrm{~mm}$ & $60 \mathrm{~mm}$ \\
\hline$[8]$ & 78.1 & Two tone & AMCW & \pm 8 & $0.4 \mathrm{~m}$ & $8 \mathrm{~mm}$ & $15 \mathrm{~cm}$ \\
\hline This & 80 & $2 \mathrm{GHz}$ & Phase modulated & \pm 7 & $0.6 \mathrm{~m}$ & $20 \mathrm{~mm}$ & $>1 \mathrm{~km}$ \\
\hline
\end{tabular}

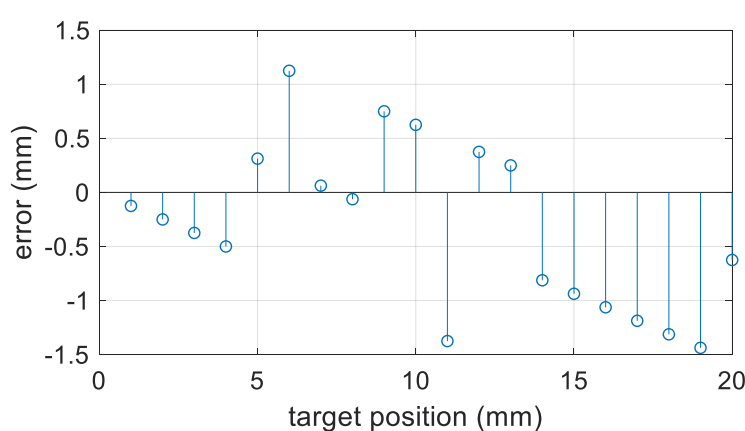

Fig. 15. Distance error vs. actual target distance after coarse distance estimation.

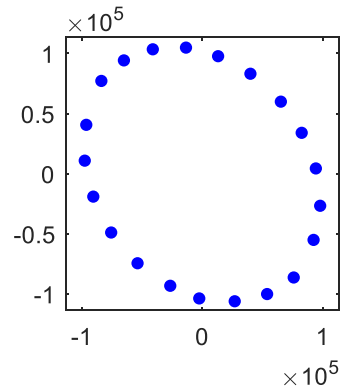

(a)

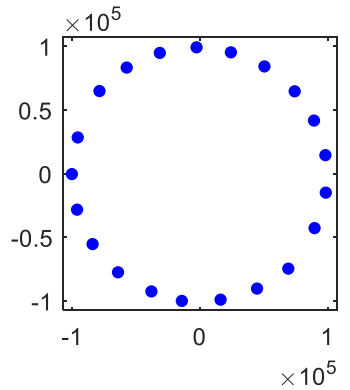

(b)
Fig. 16 The constellation points (a) in an I/Q imbalanced system, (b) after equalization.

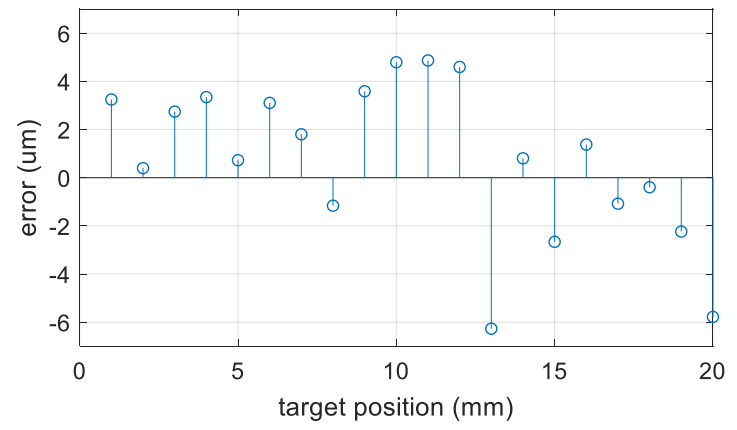

Fig. 17 Distance error vs. actual target distance.

less than the threshold. This means the proposed algorithm is capable of making a correct estimation of $\mathrm{N}$ in Eq. 4 .

\section{Fine estimation with carrier phase analysis}

Fine estimation is then performed to improve the ranging accuracy to micrometer level. As mentioned in section III. C, the phase information can be used to achieve high accuracy estimation. However, phase calibration is necessary to overcome the practical issues such as IQ imbalance of the receiver module. The received constellation has an oval shape as shown in Fig. 16 (a) that implies I/Q mismatch. The I/Q mismatch compensation as in Eq. 15 is applied with the parameter of $\alpha=0.04$ and $\beta=0.11$, the corrected constellation is shown in Fig. 16 (b).

The phase calculated by the received signal is averaged with 4000 acquired samples. After averaging, the noise power is lower which gives a better estimation of the phase information. The estimation result is shown in Fig. 17 where the estimation error is less than $7 \mu \mathrm{m}$.

\section{CONCLUSION}

In Table. I, the proposed radar system is compared with other related works. FMCW radars with phase analysis giving ranges of several micrometers was proposed in [3-5], however, these radars fail to address interference when multiple radars are closely located. Interferometer-based radar can also deliver good distance accuracy however its unambiguity distance is limited to the carrier wavelength and complicated calibration is often required [6]. With a two-tone signal, the unambiguity distance can be extended [7] and AM-modulated radar is another approach of generating two tones [9]. Both works require the shifting of center frequency in order to avoid interference. This work proposed a random phase modulated radar using PRBS code to differentiate different radars, yet high accuracy and unambiguity distance can be achieved simultaneously using proposed signal processing methods. This radar can have a high measurement repetition rate of $500 \mathrm{kHz}$ which is suitable for real-time monitoring in automatic manufacturing.

\section{ACKNOWLEDGMENT}

This work was supported in part by the Swedish Foundation for Strategic Research (SSF) under Project SE13-0020 "High Datarate Wireless Communications (100G)" and in part by the Car2TERA project which has received funding from the European Union's Horizon 2020 research and innovation programme under grant agreement No. 824962.

\section{REFERENCES}

[1] L. Xu, E. Xu and L. Li, "Industry 4.0: state of the art and future trends", Int. J. Prod. Res., vol. 56, no. 8, pp. 2941-2962, 2018.

[2] J. Lee, W. Li, J. Shen, and C. Chuang, "Multi-robotic arms automated production line," in 2018 4th International Conference on Control, Automation and Robotics (ICCAR), pp. 26-30, April 2018.

[3] S. Scherr, B. Göttel, S. Ayhan, A. Bhutani, M. Pauli, W. Winkler, J. C. Scheytt, and T. Zwick, "Miniaturized $122 \mathrm{GHz}$ ISM band FMCW radar 
with micrometer accuracy," in Proc. Eur. Radar Conf. (EuRAD), Sep. 2015, pp. 277-280

[4] M. Pauli, B. Göttel, S. Scherr, A. Bhutani, S. Ayhan, W. Winkler, and T. Zwick, "Miniaturized Millimeter-Wave Radar Sensor for High-Accuracy Applications," in IEEE Trans. Microw. Theory Tech., vol. 65, no. 5, pp. 1707-1715, May 2017.

[5] S. Ayhan, S. Thomas, N. Kong, S. Scherr, M. Pauli, T. Jaeschke, J. Wulfsberg, N. Pohl, and T. Zwick, "Millimeter-wave radar distance measurements in micro machining," in Wireless Sensors Sensor Netw. (WiSNet), Jan. 2015, pp. 65-68.

[6] F. Barbon, G. Vinci, S. Lindner, R. Weigel, and A. Klpin, "A six-port interferometer based micrometer-accuracy displacement and vibration measurement radar," in IEEE MTT-S Int. Microw. Symp. Dig., Jun. 2012, pp. 1-3.

[7] S. Lindner, F. Barbon, S. Mann, G. Vinci, R. Weigel and A. Koelpin, "Dual tone approach for unambiguous six-port based interferometric distance measurements," in IEEE MTT-S Int. Microw. Symp. Dig., Seattle, WA, 2013, pp. 1-4.

[8] F. Strömbech, Z. S. He, H. Zirath, "AMCW Radar of Micrometer Accuracy Distance Measurement and Monitoring," 2019 IEEE MTT-S International Microwave Symposium (IMS), Boston, MA, USA, 2019, pp. 1473-1475.

[9] H. Yang, and J .Chen, "Design of Costas/PSK continuous wave LPI radar signal." in International Journal of Electronics, 104.3 (2017): 404-415.

[10] R. Feger, H. Haderer, H. Jalli Ng and A. Stelzer, "Realization of a Sliding-Correlator-Based Continuous-Wave Pseudorandom Binary Phase-Coded Radar Operating in W-Band," in IEEE Trans. Microw. Theory Tech., vol. 64, no. 10, pp. 3302-3318, Oct. 2016.

[11] S. R. J. Axelsson, "Noise radar using random phase and frequency modulation," in IEEE Trans. Geosci. Remote Sens., vol. 42, no. 11, pp. 2370-2384, Nov. 2004

[12] Liu Guosui, Gu Hong and Su Weimin, "Development of random signal radars," in IEEE Trans. Aerosp. Electron. Syst., vol. 35, no. 3, pp. 770-777, July 1999.

[13] J. Tubbax, B. Come, L. Van der Perre, L. Deneire, S. Donnay and M. Engels, "Compensation of IQ imbalance in OFDM systems," IEEE International Conference on Communications, 2003. ICC '03., Anchorage, AK, 2003, pp. 3403-3407 vol.5.

[14] J. Tubbax, B. Come. L. Van der Perre, S. Donnay, M. Engels, Hugo De Man, M. Moonen, "Compensation of IQ imbalance and phase noise in OFDM systems," in IEEE Transactions on Wireless Communications, vol. 4, no. 3, pp. 872-877, May 2005.

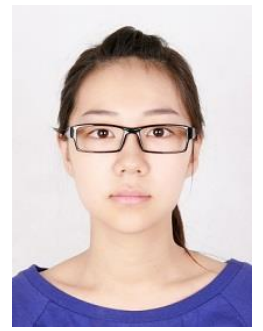

Sining An received her B.S. and M.Sc. degrees in communication and electronics engineering from the Beijing Institute of Technology, Beijing, China, in 2013 and 2016. She is currently a Ph.D. student in the Microwave Electronics Laboratory, Department of Microtechnology and Nanoscience, Chalmers University of Technology. Her research interests include high data rate communication, modulation and demodulation and high-resolution radar.

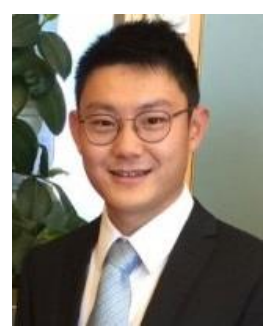

Zhongxia Simon He (M’09) received the M. Sc. degree from Beijing Institute of Technology, Beijing, China, and the $\mathrm{Ph} . \mathrm{D}$. degree from Chalmers University of Technology, Göteborg, Sweden, in 2008 and 2014, respectively. $\mathrm{He}$ is currently an Assistant Professor with the Microwave Electronics Laboratory, Department of Microtechnology and Nanoscience (MC2), Chalmers University. His current research interests include high data rate wireless communication, modulation and demodulation, mixed-signal integrated circuit design, radar, and packaging.

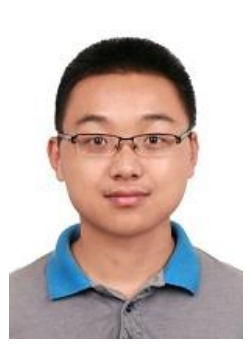

Jianguo Li received his B.S. degrees in communication and electronics engineering from the Beijing Institute of Technology, Beijing, China, in 2015. He is currently a Ph.D. student in the School of Information and Electronics, Beijing Institute of Technology. His research interests include millimeter wave communication, high precision radar, high data rate modulation and demodulation.

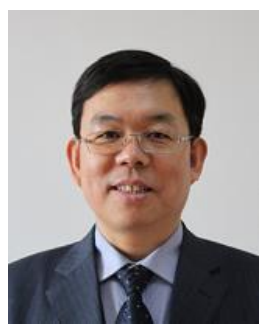

Jianping An received his $\mathrm{Ph}$. D. degree from Beijing Institute of Technology, China, in 1996. He joined the School of Information and Electronics, Beijing Institute of Technology in 1995, where he is now a full professor. He is currently the Dean of the School of Information and Electronics, Beijing Institute of Technology. His research interests are in the field of digital signal processing, cognitive radio, wireless networks, and high-dynamic broadband wireless transmission technology.

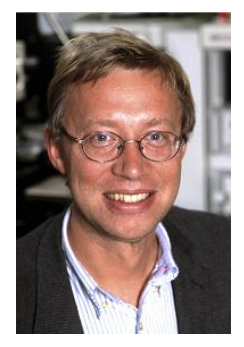

Herbert Zirath (M'86-SM'08-F'11) was born in Göteborg, Sweden, on March 20, 1955. He received the M. Sc and $\mathrm{Ph}$. D. degree in electrical engineering from Chalmers University, Göteborg, Sweden, in 1980 and 1986, respectively. From 1986 to 1996 he was a researcher at the Radio and Space Science at Chalmers University, engaged in developing a $\mathrm{GaAs}$ and $\mathrm{InP}$ based HEMT technology, including devices, models and circuits. In the spring-summer 1998 he was research fellow at Caltech, Pasadena, USA, engaged in the design of MMIC frequency multipliers and Class E Power amplifiers. He is since 1996 Professor in High Speed Electronics at the Department of Microtechnology and Nanoscience, MC2, at Chalmers University. He became the head of the Microwave Electronics Laboratory 2001. At present he is leading a group of approximately 40 researchers in the area of high frequency semiconductor devices and circuits. His main research interests include MMIC designs for wireless communication and sensor applications based on III-V, III-N, Graphene, and silicon devices. He is author/co-author of more than 530 refereed journal/conference papers, and holds 5 patents. He is research fellow at Ericsson AB, leading the development of a D-band $(110-170 \mathrm{GHz})$ chipset for high data rate wireless communication. He is a co-founder of Gotmic $\mathrm{AB}$, a company developing highly integrated frontend MMIC chip-sets for 60 $\mathrm{GHz}$ and E-band wireless communication 